\title{
Diffusive mechanism for a small mass of composite particles and new perspectives for preon models
}

\author{
Zahid Zakir $^{1}$
}

\begin{abstract}
In the diffusion of cold light gas in warm heavy gas, initially and far before the relaxation, the thermal velocities of light and heavy atoms are the same order and the light gas remains cold, and mean energies of its particles are approximately conserved. The description of such conservative diffusion is analogous to the formalism of quantum mechanics, and quantum mechanics appears as a description of such diffusion of a particle in a physical vacuum, where the diffusion coefficient is proportional to the Planck constant inverse proportional to the mass. The growth of the diffusion flux in the localization of particles leads to an increase in the osmotic pressure, which reveals the "microscopic mechanism" of the uncertainty relations and allows us to identify cases where the prohibitions imposed by them can be circumvented, in particular, to solve the mass paradox for composite particles in the preon models. If two light particles (atoms) with different masses began to diffuse at distances much greater than the mean free path, then the diffusion mechanism prevents them from further joining and as more energy must be expended to form a composite particle, as smaller the final volume of localization. However, if these particles were initially located at a distance less than the mean free path and during a time shorter than the free pass time they formed a bound state (the atoms joined in the molecule), then this composite particle (molecule) diffuses as other light particles (atoms), but with a mass slightly less than the total mass of the initial particles.
\end{abstract}

PACS: 12.60.Rc, 12.60.Nz, 03.65.Ta, 05.30.Ch, 05.40.Jc

Keywords: composite models, technicolor, quantum mechanics, conservative diffusion

\section{Content}

Introduction

1. Formation of composite particles in ordinary diffusion ...........................................................

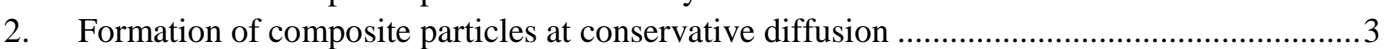

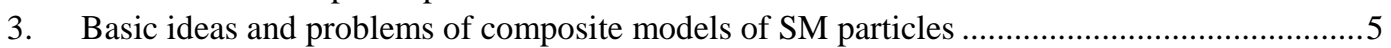

4. Models of preons with a diffusion mechanism for a small mass ............................................... 6

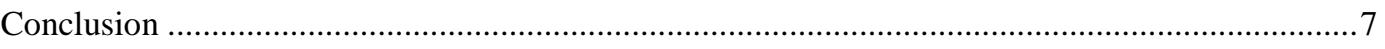

Appendix 1. Diffusion treatment of quantum mechanics ...............................................................

Appendix 2. The simplest model with two preons - the Rishon model........................................... 10

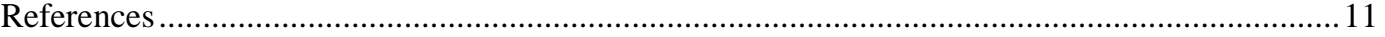

${ }^{1}$ Centre for Theoretical Physics and Astrophysics, Tashkent, Uzbekistan, zahidzakir@theor-phys.org 


\section{Introduction}

In the Standard Model (SM), which describes well the basic structures and processes of particle physics at the achieved energies, a number of particles, introduced as fundamental and structureless, is large and amounts to several tens.

However, SM does not exclude that at high energies and, correspondingly, small distances, these particles can manifest an internal structure and moreover, it is even desirable. First, the fundamental nature of the scalar field is a disadvantage of SM, since its loop contributions grow with a value of cutoff in a power-law fashion and become unacceptably large long before the Planck scale. Secondly, if the primacy of stable particles of the first generation of quarks and leptons can still be understood, it is difficult to consider as equally primary two more generations, differing only by masses.

In this connection, composite models of SM particles were developed, the subparticles of which became known under the common name of the preons, applying other names for specific models [1-3]. However, in these models a mass paradox arose - the smallness of the preon localization region leads to large kinetic energies of the preons due to the uncertainty relation, whereas the observed masses of SM particles are many orders of magnitude smaller. This means that the kinetic energy of the preons should almost completely compensated by their binding energy. But even in this case, the mass difference between the excited states must be much larger than the mass scale of the SM particles, so that the second and third generations of fermions cannot just be excited states of the first generation.

Attempts to coincide the mass paradox with quantum mechanics or to circumvent it by various ways significantly complicated the model, as a result of which the preon models ceased to evoke the former interest. Instead, this situation stimulated the development of the theories with many non-composite particles, which generated more problems than solved and led to even more complex sets of primary particles than SM.

New perspectives opened for composite models with the appearance of the diffusion treatment of quantum mechanics [4], in which quantum fluctuations are associated with the conservative diffusion of classical particles in a fluctuating vacuum. In this interpretation of quantum mechanics, a physical mechanism for gravity as thermal diffusion in physical vacuum also appears, which makes the theory of gravity as a part of the quantum theory and simplifies the problem of unifying of fields.

In this paper it will be shown that a new mechanism for the formation of small-mass composite particles, similar to the mechanism of chemical reactions between impurities, also follows from diffusive quantum mechanics. This mechanism, if it turns out to be realistic, would practically excludes the mass paradox by making composite models of SM particles consistent and thereby simplifying even more the situation with the unification of fields. In the present paper the application of this mechanism to preon models is discussed on the example of the simplest of them - the Rishon model [1-3].

Sections 1 and 2 of the paper outline the diffusion mechanism for the mass of composite particles, and in sections 3 and 4 the application of the new mechanism to the models of preons is discussed. A brief description of diffusion quantum mechanics and the Rishon model is given in Appendices 1 and 2. 


\section{Formation of composite particles in ordinary diffusion}

In physical and chemical kinetics, diffusion models of non-equilibrium processes for chemical reactions in gas mixtures have been well studied. The diffusion of electrons and ions in gases taking into account ionization and recombination is also well described by such models. They allow one to find the conditions of chemical equilibrium when impurity molecules are formed from impurity atoms (recombination) and then these molecules are dissociated [5].

The usual laws for ideal gases are applicable to the diffusion of impurities and relate partial pressures $p_{i}$ and the localization volume $V_{i}$ :

$$
p_{i} V_{i} \sim T .
$$

In a medium of a certain temperature $T$, as $V_{i}$ decreases the probability of collisions of impurity atoms with each other will increase, but then $p_{i}$ will increase too. The dissociative equilibrium conditions, when the rates of forward and backward reactions are equal, are expressed by the law of mass action, which connects the equilibrium concentrations $c_{i}$ of the initial and final components. For example, for the reaction $A+B=A B$, this law gives:

$$
c_{A} c_{B}=c_{A B} K_{c}(p, T),
$$

where $K_{c}(p, T)$ is the constant of chemical equilibrium of impurities. If the left side exceeds the right, then to achieve equilibrium either the initial concentrations should decrease or the concentration of the final bound state should increase.

In describing the random walk of two atoms of each impurity and then the random walk of the composed molecule formed by them, the concentrations must be replaced by the corresponding probability densities. As a result, we obtain a probabilistic analog of the law of mass action for the case (2):

$$
\rho_{A} \rho_{B}=\rho_{A B} K_{c}(p, T),
$$

which also follows from the definition of conditional probabilities.

All this concern ordinary (dissipative) diffusion, when impurities are in thermal equilibrium with the medium (solvent) and the mean kinetic energies of the impurity and solvent molecules are equal. In the next section, we will consider the case when the mass of the impurity atoms are sufficiently small than the mass of the medium atoms, and far before relaxation, not the kinetic energies but the thermal velocities are equal, which radically changes the whole picture, since the diffusion turns out to be conservative.

\section{Formation of composite particles at conservative diffusion}

Conservative diffusion is realized by diffusion of a cold light gas in a warm heavy gas in a short time interval at the beginning of the relaxation, when the thermal velocities of light and heavy atoms are close, the light gas remains cold and the mean energy of its particles is approximately conserved. The theory of such diffusion and its application to the quantum theory are summarized in Appendix 1. Quantum mechanics turns out to be a description of the conservative diffusion of classical particles in a vacuum with the diffusion coefficient $D=\hbar / 2 m[4]$.

In the diffusion interpretation, quantum mechanical phenomena appear as consequences of ordinary gas laws and diffusion phenomena, and it is unusual only the conservation of the mean energy of diffusing particles, which is realized even in ordinary 
gases under specific conditions and in a short time interval. In particular, the uncertainty relation is a consequence of gas laws for impurities similar to (1):

$$
p_{i} V_{i} \sim T_{i},
$$

where the temperatures of impurities $T_{i}$ are now different and much lower than the temperature of the medium $T_{i} \ll T$. Particles act as "impurities" in a fluctuating vacuum with a certain temperature and the diffusion flux grows with decreasing of their localization volume. Such explaining of the "microscopic mechanism" of the uncertainty relations makes it possible to reveal also cases in which their limitations do not work under certain conditions, which then solves the mass paradox.

Two examples of such a legal "circumvention" of a certain rigid prohibition are given by relativistic kinematics and chromodynamics. In the first case, the unrestricted growth of the energy of a particle with a nonzero mass at approaching the speed of light forbids such particles from reaching the speed of light, but if a particle is born with zero mass, then this prohibition does not act on it and it moves at the speed of light. In the second case, the unbounded growth of the hadrons interaction force as they approach each other is removed when taking into account the hadron size, inside which chromodynamics with asymptotic freedom acts and the interaction forces decrease by distance between quarks.

In the case of diffusion in gases, there is a direct analogy with the last example, since the presence of regions with mean free path $l_{D}$ separates compound objects and processes into two groups. The first is structures and processes with characteristic dimensions $l \gg l_{D}$, where gas laws operate, and the latter - with dimensions $l \ll l_{D}$ where the trajectories of atoms between collisions are classical and there is "asymptotic freedom" from gas laws.

In the admixture of light gases in heavy gas, chemical reactions between impurities must have time to occur in times much shorter than the mean free pass time in the gas. If two impurity atoms have time to merge before next collision with the molecules of the medium, and the binding energy exceeds the thermal energy of the latter, then the new molecule will not quickly decay. Under these conditions, the formed molecules further diffuse similar to the impurity atoms, but with a larger mass. Since the masses of the impurity atoms were much smaller than the mass of the solvent gas molecules, the new molecules of the mixture also remain light compared to the solvent molecules.

So, if two light particles (atoms) having masses $m_{1}$ and $m_{2}$ began to diffuse in heavy gas at distances sufficiently larger $l_{D}$, then the diffusion mechanism prevents their further approach and for the formation of a composite particle, it is necessary to expend as more energy, as smaller the volume of localization. However, if the same two particles at the beginning of the process were at distance smaller $l_{D}$, and in time less than the free pass time $\tau_{D}$, they formed a compact bound state (the atoms formed a molecule) having small scale factor $l_{s u b} \ll l_{D}$. Then this compound particle (molecule) diffuses with high probability practically as other light particles (atoms), but with a mass of order of the total mass $m_{1}+m_{2}$ . The probability of the decay (dissociation) of the composite particle in the future is as small as the bond energy is larger than the thermal energy in the medium.

The diffusional nature of quantum fluctuations, therefore, leads to a new possibility of forming composite particles in quantum mechanics, where the particles are classical and only diffuse in a vacuum. Two classical "point" particles may approach at a very small 
distance $l_{\text {sub }}$ and may have time to form a bound state. Despite the smallness of the probability of their collisions, if only collisions are taken into account, then the probability of successful connections will be sufficiently large, and the probability of formation of composite particles could be calculated by a relationship like (3).

In the case of conservative diffusion, however, it is not the probabilities of alternative and simultaneous events should be added and multiplied, but their amplitudes. Therefore, instead of the relation (3), there will be a definition of the transition probability for conservative diffusion (familiar from quantum mechanics):

$$
\langle A B \mid A, B\rangle=\tilde{K}_{c}(p, T) .
$$

The equilibrium constants $\tilde{K}_{c}$, of course, depend on the nature of the forces that form the bound state.

If, in this case, the binding energy $U_{s}$ is greater in magnitude than the energy of the vacuum fluctuations at these distances

$$
\left|U_{s}\right|>\hbar c / l_{\text {sub }}
$$

then the formed (also classical) composite particle will be stable. Its mass $m_{12}$ in this case will be less than the sum of the masses of the constituent particles by the magnitude of the mass defect

where $\Delta m=\left|U_{s}\right| / c^{2}$.

$$
m_{12} \simeq m_{1}+m_{2}-\Delta m,
$$

Here we shall not go into the details of this phenomenon, confining ourselves to the very fact of the existence of such a possibility in principle, and further in Section 4 we shall consider some consequences of this fact for composite models of SM particles.

\section{Basic ideas and problems of composite models of SM particles}

In the SM there are a number of problems, at trying to solve of which the composite models arose. A scalar field leads to loop contributions growing with a cutoff of energy in a power-law manner, and therefore the scalar particles of SM are unlikely to be primary. The first composite models of scalar particles were models of technicolor. These models increased both the number of primary fermions (adding technical fermions) and gauge bosons (technicolor), which made them much more complicated than SM.

But the introduction of technifermions makes it possible to build fermions of SM from them, which not only greatly simplifies the technicolor models, but it can also solve the problem of repeating the generations of quarks and leptons. Therefore, the logical step was the creation, by analogy with the technicolor, of models where both scalars and all the fermions of SM are composite. However, in these models the number of gauge bosons will still be larger than in SM, which complicates the unification of fields.

As a result, the next logical step was the construction of not only scalars and fermions from subparticles - preons - but also gauge bosons of SM, which greatly simplified both the composition of the primary particles and the unification of the fields. The creation of such radical models completed the first stage of development of composite models, when the proposed models were free from the main problems of SM, being much simpler than SM.

At the same time, each of the models had a number of its own problems, and there was one common problem for all of them - the mass paradox. The simplest, logical and elegant of such models is the Rishon model [1,2], a brief description of which is given in Appendix 2. 
The Rishon model is extremely economical - all the SM particles consist of only two kinds of preons - rishons $T$ and $V$ with spin $1 / 2$ and electric charges $Q=(1 / 3,0)$, as well as their antiparticles. Quantum numbers of bound states of three rishons exactly reproduce the quantum numbers of fermions of the first generation of SM, and the same states with the addition of one of the pairs $V \bar{V}$ or $T \bar{T}$ lead to two more generations. The dynamics underlying the model is subject to refinement and there are different versions [3], but the main difficulty of the model was associated with the mass paradox. We will consider the solution of the mass paradox following from the diffusion approach on the example of this model.

The solution of the mass paradox completes the second stage in the development of composite models when their consistency from the theoretical point of view is reached and the third stage begins when the theory should answer the questions of dynamics, and the experiment will indicate the scales of distances and energies where the preon substructure reveals. These scales are in a large interval of distances $10^{-18} \div 10^{-33} \mathrm{~cm}$ and energies $10^{3} \div 10^{19} \mathrm{GeV}$ and the search for the substructure of SM particles in this region and verification of predictions of the dynamic models become further the main trend in particle physics.

\section{Models of preons with a diffusion mechanism for a small mass}

The mass paradox arises for two reasons. The first reason is the smallness of the binding energy to compensate the kinetic energy of the subparticles. The second reason is the application to the composite models of the standard formalism of quantum mechanics, including the uncertainty relations, without taking into account the details of the "microscopic mechanism" of quantum fluctuations.

In the diffusion treatment, which deduces quantum-mechanical laws from a specific "microscopic mechanism" - the conservative diffusion of classical particles in a fluctuating vacuum - the mass paradox does not arise under certain conditions, which has already been discussed in Section 2. Let us now clarify these conditions and their consequences for composite models, taking into account the specifics of preons and their supposed interactions.

The main conditions for the formation of small-mass composite particles, even before the characterization of the interactions of the preons, are following:

1) the masses of the preons must be sufficiently small, apparently they should even be massless so that they can form a massless photon and very light neutrinos;

2) the preons must be considered as "point" particles in the sense that their dimensions are much smaller than the radius of the constituent particles;

3) the dimensions of the composite particles must in turn be much less than the "mean free path" for diffusion of preons in the physical vacuum;

4) the binding energy of the preons should exceed the energy of vacuum fluctuations at the radius of the compound particle (6).

The lifetime of a composite particle is as greater as the binding energy is greater than the "thermal energy" in the physical vacuum. Since in the diffusion interpretation the rest energy of the particles is associated with their thermal energy in the physical vacuum [4], it follows that as smaller the rest mass of the composite particle, as more stable it is. The limiting expression of this property would be the assertion that the composite particles of the smallest mass for a given charge and spin are stable. This is confirmed by the stability of the fermions of the first generation, as well as the photon and, possibly, the gluons. 


\section{Conclusion}

In the framework of standard quantum mechanics, where the localization of particles is limited by the uncertainty relation, the mass paradox in the composite quark and lepton models is practically unsolved, and therefore the prospects for building of consistent models are very vague, as manifested in stagnation in this field of research over the past more than three decades.

Within the framework of the diffusion interpretation of quantum mechanics, which is the next step in understanding the mechanisms of quantum phenomena, where the latter are explained from the conservative diffusion of classical particles in a physical vacuum, the uncertainty relations are not an absolute limitation and, therefore, new prospects for a natural resolution of the mass paradox are opened.

The new mechanism is an analog of chemical reactions between mixtures in gases that occur at distances smaller than the mean free path and depend little on the diffusion mechanisms acting at larger distances between the atoms of the mixture. Therefore, at distances much shorter than the "mean free pass time" of the subparticles in the physical vacuum, they can form composite systems with a total mass much less than that follows from the uncertainty relations.

Thus, the diffusional nature of quantum fluctuations leads to a new mechanism for the formation of small-mass composite particles, when their mass may not be of the order of $\sim \hbar / c l_{s u b}$, but of the order of the sum of the effective masses of the preons, i.e. $m_{1}+m_{2}$.

\section{Appendix 1. Diffusion treatment of quantum mechanics}

The diffusion of a cold light gas in a warm heavy gas at the initial stage before relaxation, as it turned out, qualitatively differs from the usual dissipative diffusion [4]. In the ideal gas model, the mean energy of light particles is approximately conserved here and the process is very close to conservative diffusion. At colliding with a heavy particle of the medium in the rest system of the latter, it mainly changes the direction of the velocity of the light particle, and the change in the value of the velocity is very small. In the rest system of the gas, the fluctuations of the light particle velocity is of order $\delta v_{D} \sim V=(6 k T / M)^{1 / 2}$, where $V$ is the mean thermal velocity of the heavy particles, $T$ is the temperature of the medium, $M$ is the mass of the heavy particles, and $k$ is the Boltzmann constant. The trajectories of light atoms consist of free paths between collisions, and their mean kinetic energy is conserved at a large number of collisions $n_{D} \sim M / m$, where $m$ is the mass of a light particle. In the first approximation, the process is statistically reversible in time

In the period of conservativity (i.e., before relaxation), the ensemble of light particles is not in thermal equilibrium with the medium. In contrast to the Lorentz gas, where the thermal energies of the atoms of the light and heavy gas are equal, in our case, only their thermal velocities are of the same order. Therefore, the thermal energy of the light particles, as well as the temperature of a light gas $T_{l}$, will be much less than that of a heavy gas $T_{l} \ll T$.

At such conservative diffusion, averaging over the ensemble of light particles at each moment $t$ for the mean free path, we determine the mean length $l_{D}$ and mean time $\tau_{D}$, velocity $v_{D}=l_{D} / \tau_{D}$, momentum $p_{D}=m v_{D}$, kinetic energy $E_{D}=m v_{D}^{2} / 2$, and 
abbreviated action $S_{D}=p_{D} l_{D}$. In statistical mechanics, an element of the phase volume $\Delta \Gamma=\Delta p \Delta x$ is introduced where the particle is in the time interval $\Delta t$. In our case, there is a distinguished value of the elementary phase volume:

$$
\Delta \Gamma=p_{D} l_{D}=m l_{D}^{2} / \tau_{D}=\Gamma_{D},
$$

coinciding with $S_{D}$. The definition of the diffusion coefficient $l_{D}^{2}=2 D \tau_{D}$ then gives:

$$
\Gamma_{D}=2 m D, \quad D=\Gamma_{D} / 2 m .
$$

The theory of ordinary dissipative diffusion in an equilibrium mixture for the random walk of one light particle leads to the formalism of Brownian motion. For conservative diffusion, the hydrodynamic formalism turns out to be natural. Since the trajectory of a particle between collisions is classical, the drift momentum $\mathbf{p}_{\mathbf{v}}=m \mathbf{v}$, where $\mathbf{v}$ is the drift velocity, is expressed in terms of the action function $S(x, t)$ as:

$$
m \mathbf{v}(x, t)=\nabla S(x, t) .
$$

Let the concentration of light particles $c_{D}$ inside the region of space $\Delta V$ be greater than outside of $\Delta V$. At small intervals of time, the number of light particles that leave on $\Delta V$ averagely is larger than those entering it, i.e. here there is a diffusion flux $\mathbf{j}_{D}=\mathbf{u} c_{D}$ of light particles from $\Delta V$ to the outer region, where $\mathbf{u}$ is the velocity of diffusive flow. In an isothermal medium ( $T=$ const. ), in the first approximation, this flux is proportional to the gradient of concentration and directed in the opposite direction:

$$
\mathbf{j}_{D}=\mathbf{u} c_{D}=-D \cdot \nabla c_{D}, \quad \mathbf{u}=-D \frac{\nabla c_{D}}{c_{D}}=-D \frac{\nabla n_{D}}{n_{D}},
$$

where $n_{D}$ - is the density of the number of light particles. If we describe only one light particle, then in Eq. (11) instead of $n_{D}$ it is presented the probability density $\rho(x, t)$, which is normalized to unity, and, due to the conservation of probability, it satisfies the continuity equation:

$$
\frac{\partial \rho}{\partial t}+\nabla \cdot(\mathbf{v} \rho)=0
$$

The relation for $\mathbf{u}$ in (11) and the properties of $\mathbf{u}$ then are described by:

$$
\mathbf{u}=-D \cdot \frac{\nabla \rho}{\rho}, \quad \overline{\mathbf{u}}=\int \mathbf{u} \rho d^{3} x=0, \quad \int \mathbf{u}^{2} \rho d^{3} x \neq 0 .
$$

The momentum $\mathbf{p}_{u}=m \mathbf{u}$ satisfies the uncertainty relation (at $\overline{\mathbf{x}}=0$ ):

$$
\sqrt{\overline{\mathbf{p}_{u}^{2}} \cdot \overline{\mathbf{x}^{2}}} \geq\left|\overline{\mathbf{p}_{u} \cdot \mathbf{x}}\right|=m\left|\int \mathbf{u} \cdot \mathbf{x} \rho d^{3} x\right|=m D\left|\int \nabla \rho \cdot \mathbf{x} d^{3} x\right|=\frac{\Gamma_{D}}{2} .
$$

The Hamiltonian, taking into account (10) and (13), acquires the form:

$$
H=\int\left(\frac{\mathbf{p}_{\mathbf{v}}^{2}}{2 m}+\frac{\mathbf{p}_{\mathbf{u}}^{2}}{2 m}+V\right) \rho d^{3} x=\int\left(\frac{1}{2 m}(\nabla S)^{2}+\frac{\Gamma_{D}^{2}}{8 m}\left(\frac{\nabla \rho}{\rho}\right)^{2}+V\right) \rho d^{3} x,
$$

where the canonical pair is $S(x, t)$ and $\rho(x, t)$, and the Poisson brackets for it have the form:

$$
\{A, B\}=\int\left(\frac{\delta A}{\delta \rho} \frac{\delta B}{\delta S}-\frac{\delta B}{\delta \rho} \frac{\delta A}{\delta S}\right) d^{3} x, \quad\left\{\rho(x, t), S\left(x^{\prime}, t\right)\right\}=\delta\left(x-x^{\prime}\right) .
$$

Corresponding canonical equations: 


$$
\frac{\partial \rho}{\partial t}=\{\rho, H\}=\frac{\delta H}{\delta S}, \quad \frac{\partial S}{\partial t}=\{S, H\}=-\frac{\delta H}{\delta \rho} .
$$

give the continuity equation (12) and the Hamilton-Jacobi equation:

$$
\frac{\partial S}{\partial t}+\left(\frac{(\nabla S)^{2}}{2 m}+V\right)-\frac{\Gamma_{D}^{2}}{8 m} \frac{\Delta \sqrt{\rho}}{\sqrt{\rho}}=0,
$$

The probability density $\rho$ enters to this system of equations nonlinearly and for two alternatives we have $\rho_{12} \neq \rho_{1}+\rho_{2}$, but these equations are linearized under the canonical transformation to a canonical pair $\psi_{1}, \psi_{2}$, which can be represented also as a complex probability amplitude:

$$
\psi=\psi_{1}+i \psi_{2}=\sqrt{\rho} \exp \left(i S / \Gamma_{D}\right) .
$$

The equations (12)-(18) then transform to the Schrödinger equation for $\psi(x, t)$ :

$$
i \Gamma_{D} \frac{\partial \psi}{\partial t}=\left(-\frac{\Gamma_{D}^{2}}{2 m} \Delta+V\right) \psi .
$$

Here there is a linear superposition of states: $\psi=c_{1} \psi_{(1)}+c_{2} \psi_{(2)}+\ldots$ and therefore, in classical conservative diffusion, there added not the probabilities of alternatives, but their probability amplitudes. The physical meaning of the wave behavior is the periodic repetition along the trajectory of free pass sections with the mean length $l_{D}$, and also in the presence of an elementary phase volume $\Gamma_{D}$ associated with them.

Thus, conservative diffusion in classical systems under the conditions indicated above is described by a mathematical formalism of quantum mechanics with replacement $\hbar \rightarrow \Gamma_{D}$. The quantum mechanics turns out to be only a special case of classical conservative diffusion at $\Gamma_{D}=\hbar$. "Quantization" thus reduces to the fact that the classical particle, which in the nonrelativistic theory in the classical (empty) space and the force potential $V$ would have energy $\mathbf{p}_{\mathbf{v}}^{2} / 2 m+V$, being placed in the physical vacuum fluctuates, and to its "momentum" and energy are added "thermal" and diffusion contributions

The state of a free particle corresponds to a plane wave $\psi \sim \exp [i(\mathbf{p x}-E t) / 2 m D]$, where $S(\mathbf{x}, t)=\mathbf{p x}-E t$ and $\rho=$ const., so that in this case $\mathbf{u} \sim \nabla \rho=0$ and there is no diffusional part of the nonrelativistic momentum $\mathbf{p}_{u}=0$, and therefore $E_{u}=0$. For a bound state $S(t)=-E t, \rho(x)$ ensures the localization of the particle in the vicinity of the center of mass with $\mathbf{u}^{2}>0$, and $U_{u}=\mathbf{p}_{u}^{2} / 2 m$ is the energy that was expended (by the box walls or the force field) to localize the particle in the given region. Therefore, as smaller the region of localization of a particle, as larger the localization energy $U_{u}$, which expresses the uncertainty relation (14).

In relativistic theory, there is a constant addition to the energy of any particle of finite mass - a rest energy $E_{0}=m c^{2}$, whose physical meaning was unclear. In the diffusion treatment, it turns out to be the thermal energy $\sim k T_{l}$ of the particles in the physical vacuum.

When many particles are diffused in compact region, an appreciable fraction of the energy of the vacuum is expended to their fluctuations, which reduces the local energy density of the vacuum and there a "temperature gradient" arises. This leads to the thermal diffusion [4], which has all the properties inherent in gravity, i.e. gravitation in diffusion quantum mechanics arises naturally as a manifestation of conservative thermal diffusion in the vacuum. 


\section{Appendix 2. The simplest model with two preons - the Rishon model}

The rishon model [1, 2] is the simplest in the sense of the number of preons (only two preons $T, V$ and their antiparticles $\bar{T}, \bar{V}$ ). Both preons (called rishons) are fermions with spin $J=1 / 2$ and differ from each other mainly by electric charge: $T$ and $\bar{T}$ are charged ( $\left.Q_{T}=1 / 3, Q_{\bar{T}}=-1 / 3\right)$, while $V, \bar{V}$ are electrically neutral $\left(Q_{V}=Q_{\bar{V}}=0\right)$. Their masses are considered small.

In this model, all SM particles, both fermions and bosons, composite -fermions are 3preon states, and gauge bosons are 6-preon states (in the original version of the model [1, 2]). Attempts to describe the dynamics by analogy with chromodynamics have sufficiently complicated the model [3] and therefore we confine ourselves to the simplest version of the model, leaving for the future clarification of the dynamics, which may turn out to be unusual.

The simplest composite fermion can be constructed from three preons. Of the eight possible combinations, two correspond to leptons, and six to two quarks with three colored states. The second and third generations of quarks and leptons can correspond to either the excited levels of the first generation, or, more likely, differ from them by the presence of pairs $V \bar{V}$ and $T \bar{T}$ [3]. The colors of the quarks arise from the difference in the three locations of one of the preons in the quark, although it is not yet clear how they differ. Table 1 gives the preon composition of the fermions SM.

Here, the comparison of the second and third generations of states with one of the pairs $V \bar{V}$ and $T \bar{T}$ is conditional and given for an example, since depending on the details of the dynamics and the contribution to the mass, these two columns can change places.

The conservation of the difference between the numbers of the preons and antipreons of each species leads to two conserved quantum numbers for composite SM particles:

$$
n(T)-n(\bar{T})=3 Q, \quad n(V)-n(\bar{V})=3 Q-3(B-L),
$$

where $B, L-$ are the numbers of baryons and leptons.

Table 1. The preon composition of fermions.

\begin{tabular}{|c|c|c|c|c|c|c|}
\hline $\mathrm{Q}$ & \multicolumn{2}{|c|}{ 1- generation } & \multicolumn{2}{|c|}{ 2- generation } & \multicolumn{2}{c|}{ 3-generation } \\
\hline+1 & $e^{+}$ & $T T T$ & $\mu^{+}$ & $T T T \bar{V} V$ & $\tau^{+}$ & $T T T \bar{T} T$ \\
\hline \multirow{4}{*}{$+2 / 3$} & $u_{1}$ & $T T V$ & $c_{1}$ & $T T V \bar{V} V$ & $t_{1}$ & $T T V \overline{T T}$ \\
& $u_{2}$ & $T V T$ & $c_{2}$ & $T V T \bar{V} V$ & $t_{2}$ & $T V T \bar{T} T$ \\
& $u_{3}$ & $V T T$ & $c_{3}$ & $V T T \bar{V} V$ & $t_{3}$ & $V T T \bar{T} T$ \\
\hline \multirow{4}{*}{$+1 / 3$} & $\bar{d}_{1}$ & $V V T$ & $\bar{s}_{1}$ & $T V V \bar{V} V$ & $\bar{b}_{1}$ & $T V V \overline{T T}$ \\
& $\bar{d}_{2}$ & $V T V$ & $\bar{s}_{2}$ & $V T V \bar{V} V$ & $\bar{b}_{2}$ & $V T V \bar{T} T$ \\
& $\bar{d}_{3}$ & $T V V$ & $\bar{s}_{3}$ & $V V T \bar{V} V$ & $\bar{b}_{3}$ & $V V T \bar{T} T$ \\
\hline 0 & $v_{e}$ & $V V V$ & $v_{\mu}$ & $V V V \bar{V} V$ & $v_{\tau}$ & $V V V \overline{T T}$ \\
\hline
\end{tabular}

In

each

generation of fermions the total number of preons is equal to the total number of antipreons ( $6 T+6 V+6 \bar{T}+6 \bar{V}$ ). Moreover, at the level of the preons, matter and antimatter are equally distributed in the universe, since the electron-proton pair, as well as the neutron, contains an equal number of preons and antipreons.

The states of the preon-antipreon pair $V \bar{V}, T \bar{T}$ with $J=0$ can correspond to the scalar constituent particles, and with $J=1$ - to the vector particles. Two pairs of $J=1$ can 
form both new scalars and 4-preon states with $J=2: V V \bar{V} \bar{V}, T V \bar{V} \bar{T}, V T \bar{T} \bar{V}$ and $T T \bar{T} \bar{T}$ being an analog of the graviton.

The states of the three preon-antipreon pairs correspond to the vector gauge bosons of SM. The preon composition of SM bosons is given in Table 2.

Table 2 . The preon composition of bosons of SM.

a) bosons of the electroweak theory b) gluons

\begin{tabular}{|l|l|}
\hline$H_{V}$ & $V \bar{V}$ \\
\hline$H_{T}$ & $T \bar{T}$ \\
\hline
\end{tabular}

\begin{tabular}{|c|c|}
\hline$W^{+}$ & $T T T V V V$ \\
\hline$W^{-}$ & $\bar{T} \bar{T} \bar{T} \bar{V} \bar{V} \bar{V}$ \\
\hline$A^{0}$ & $V V V \bar{V} \bar{V} \bar{V}$ \\
\hline$B$ & $T T T \bar{T} \bar{T} \bar{T}$ \\
\hline
\end{tabular}

\begin{tabular}{|l|l|l|l|}
\hline$G_{12}$ & $T T V \bar{T} \bar{V} \bar{T}$ & $\tilde{G}_{12}$ & $V V T \bar{V} \bar{T} \bar{V}$ \\
\hline$G_{21}$ & $T V T \bar{T} \bar{T} \bar{V}$ & $\tilde{G}_{21}$ & $V T V \bar{V} \bar{V} \bar{T}$ \\
\hline$G_{23}$ & $T V T \bar{V} \bar{T} \bar{T}$ & $\tilde{G}_{23}$ & $V T V \bar{T} \bar{V} \bar{V}$ \\
\hline$G_{32}$ & $V T T \bar{T} \bar{V} \bar{T}$ & $\tilde{G}_{32}$ & $T V V \bar{V} \bar{T} \bar{V}$ \\
\hline
\end{tabular}

Photons and $Z^{0}$ are formed from $A^{0}$ and $B$ by mixing with the Weinberg angle $\theta_{W}$. While the parity violation mechanism in weak interactions is not clear, the model allows us to calculate $\theta_{W}$ and give $\sin ^{2} \theta_{W}=1 / 4$ at the level of substructures close to the observed value 0.23 at the level of composite particles.

The gluons reconstruct the preons in the quark, transferring the states VTT to TVT, $V V T$ to $V T V$ and etc., i.e. they act to color states only.

\section{References}

1. Harari H. (1979) Phys. Lett. 86B, 83.

2. Shupe M.A. (1979) Phys. Lett. 86B, 87.

3. Harari H., Seiberg N. (1981) Phys. Lett. 98B, 269.

4. Zakir Z. (2014) Theor. Phys., Astrophys. and Cosmol. 9(2) 54, doi: 10.9751/TPAC. 4960-038.

5. Landau L.D., Lifshitz E.M. (1980) Statistical Physics. Pergamon. 